\title{
Japanese Encephalitis in Children admitted at Patan Hospital
}

\author{
Shrestha SR ${ }^{1,}$ Awale $\mathrm{P}^{2}$, Neupane $\mathrm{S}^{3}$, Adhikari N${ }^{4}$, Yadav BK \\ ${ }^{1}$ Dr, Sita Ram Shrestha. MBBS. DCH MD. Paediatrician and Sr Registrar Department of General Practice and \\ Emergency Medicine. ${ }^{2}$ Dr Pankaj Awale. MBBS. MD.General Practitioner. Department of Genereal Practice and \\ Emergency Medicine, ${ }^{3}$ Dr Sukadev Neupane. MBBS. MHCM. WHO/IPD Nepal ${ }^{4}$ Dr. Neelam Adhikary. MBBS. MD. \\ Chief of Pediatric Services, Department of Pediatrics. ${ }^{5}$ Dr BK Yadav MBBS, MD. Consultant. Department of GP and \\ Emergency Medicine. All from Patan Hospital.
}

Address for Correspondence: sitaram2051@gmail.com

\begin{abstract}
This is a hospital based retrospective study, which was done in Pediatric ward of Patan hospital. Study period was one and half year (from Srawan 2063 to 2064 poush).Data were taken from discharge book of Pediatric ward, from the record section of this hospital, and from JE surveillance office, WHO, Kathmandu. All children from 1 month to 14 years, who were admitted in Pediatric ward with symptoms of Meningitis, Meningoencephalitis and Encephalitis were included in this study and patients more than 14 years of age and symptoms not suggestive of meningitis, meningoencephalitis or encephalitis were excluded from the study. Headache; vomiting and fever were the chief complaints of patients. Two patients died during study period. There were 16 patients with serologically confirmed Japanese encephalitis.
\end{abstract}

Key words: Japanese encephalitis virus (JEV), arthropod borne disease, Acute encephalitis syndrome (AES).

\section{Introduction}

Japanese encephalitis virus (JEV), arthropod borne pathogen infections can be found throughout the temperate and tropical zones of Asia. ${ }^{2}$ Approximately 3 billion people and $60 \%$ of the world's population live in JE-endemic regions and there are approximately, 50000 cases and 15,000 deaths per annum were notified from wide geographical range ${ }^{3}$. In endemic areas annual incidence ranges from $10-100$ per 100000 populations $^{4}$ The virus was isolated for the first time in the world from a post mortem human brain in Japan in 1933AD, although descriptive accounts of the disease date back to late $1800 \mathrm{AD} .{ }^{1}$ In 1954 , it was shown that virus could also infect pigs, bovines, dogs and sheep. ${ }^{5}$ Basically, $\mathrm{JE}$ is a zoonotic disease maintaining JEV in nature by bird mosquito bird and pig mosquito pig cycles. ${ }^{6}$ Pigs are amplifiers of JEV. Bats can also carry the virus for longer period of time. Human beings are only incidental hosts forming a dead end.

Agrowing number of cases of Japanese Encephalitis have been seen in horses in China and humans in India, Nepal, Philippines Sri Lanka, Northern Thailand, Vietnam, and Myanmar. ${ }^{1} \mathrm{JEV}$ is the most common documented cause of viral encephalitis in Cambodia ${ }^{7}$.
Rise in population density, deforestation and increasing irrigation of agricultural areas may contribute to the rise in JE incidence each year JEV infections were occasionally found in Indonesia and Northern Australia but never found in United States. ${ }^{1}$ In recent years, JE is rare in Japan due to JE virus vaccination, use of agricultural pesticides and controlled pig farming. 1,8 The occurrence of JE has also been reported less in Korea, China and Taiwan due to JE virus vaccination of children.

Japanese Encephalitis (JE) known "a plague of the Orient" continues to be the most common human epidemic encephalitis in the world. About one third of the patients die and half of the survivors have residual sequalae. ${ }^{3}$ The major burden of this disease is in children. Diagnosis depends on a high degree of clinical suspicion and confirmation by serology or culture: Cerebrospinal fluid (CSF) analysis, computered tomography and magnetic resonance imaging are also important.

Most studies of the clinical features of JE have been based on information collected during epidemics and during which diagnosis was confirmed only a small number. ${ }^{9}$ Even during epidemics, many patients 
might be suffering from other causes of acute febrile encephalopathy. ${ }^{10}$

This disease was first recorded in Nepal in 1978 as an epidemic in Rupandehi district of the western development region and Morang of the eastern region. At present this disease is endemic in 24 districts of the country. Although JE as found endemic mainly in tropical climate areas, existence and proliferation of encephalitis causing viruses in temperate and cold climates of hills and valleys are possible. ${ }^{11}$ More than $50 \%$ of morbidity and $60 \%$ mortality occur in the age group below 15 years. Upsurge of cases take place after the rainy season (monsoon). Cases start to appear in the month of April-May and reach its peak during late August to early September and decline from September. ${ }^{11}$

Japanese Encephalitis is demonstrated to be a significant public problem throughout Asia. It primarily affects children between the ages of one and 15 years. Of those who contract the diseases, approximately 70 $\%$ either die or are left with a long term neurological disability. JE vaccines due to various causes have not been able to meet the needs of developing country's health systems. ${ }^{12}$

World studies reports mortality rate due to JE ranges from $23 \%$ to $36 \%$ and $18 \%$ of cases end up with complications. ${ }^{13,14,15}$ Some studies say that infection may also result in residual sequelae in $30 \%-60 \%$ of cases. ${ }^{16}$ Limited data has also indicated that JE acquired during the first or second trimesters of pregnancy can cause intrauterine infection and miscarriage. ${ }^{18}$

The main objectives of this study are to study the most common symptoms of presentation of Japanese encephalitis among admitted children at Patan Hospital, the prevalence of disease in different months of year and to know the serologically confirmed cases among AES.

\section{Method of Study}

This is a hospital based retrospective study, done in Pediatric ward of Patan hospital. Ninty patients were included in this study and the study period was one and half year (from Srawan 2063 to 2064 poush i.e. July 2006 to December 2007). Data was extracted from the discharge book of Pediatric ward, record section of OPD, JE surveillance office which was then analyzed. Inclusion criteria: All children from 1 month to 14 years, admitted in Pediatric ward with symptoms of Meningitis, Meningoencephalitis and encephalitis. Exclusion criteria: All children more than 14 years of age, Children with symptoms not suggestive of meningitis or encephalitis.

\section{Results}

There were a total of ninty (90) Acute Encephalitis like Syndrome (AES) out of which sixteen (16) had JE confirmed by serology and culture. It was seen that JE had decreased from the year 2007 as compared to 2006. Even though a female preponderance was noted in the initial years of life, it remained equal from the age of 5 years onwards. Symptomatically fever $(n=16)$ was the most common symptom of patients followed by vomiting $(n=8)$ and headache $(n=5)$. Other symptoms such as loss of consciousness, altered level of sensorium and seizures were also noted. Annual pattern of JE showed that most of the cases were observed during August and September months. Out of the total sixteen patients two died during study period the case fatality rate being $12.5 \%$.

Fig. 1: Showing the total (AES) Patients and Laboratory Results Confirmed JE Patients.

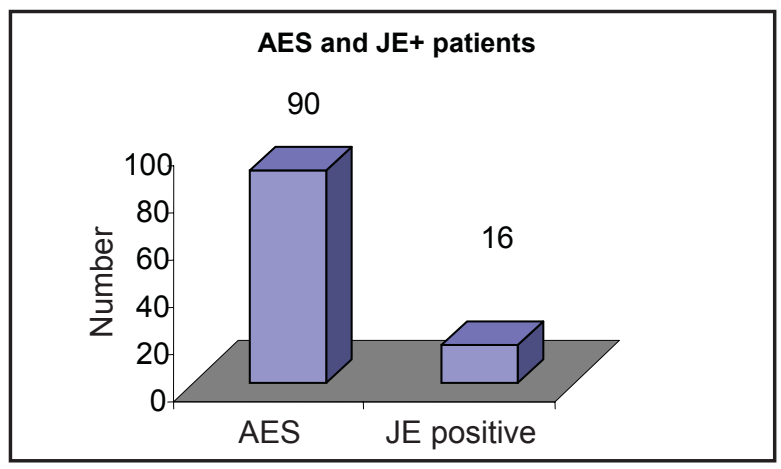

Fig 2: Showing that JE in different age years.

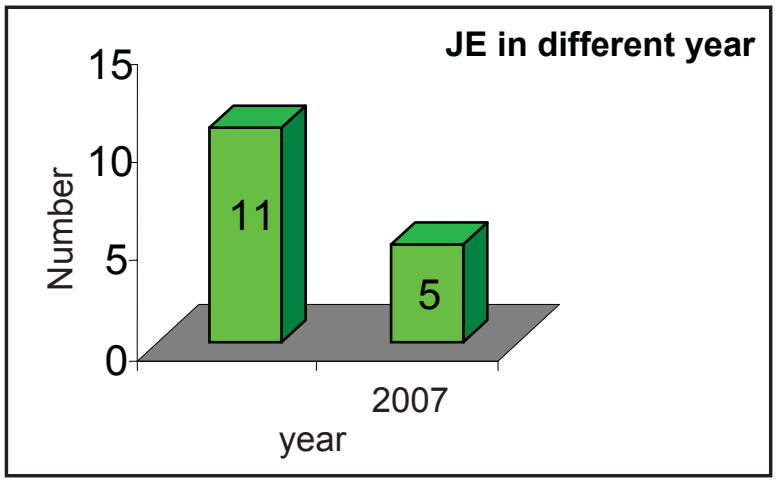

Fig. 3: Showing the Age and Sex distribution of JE patients.

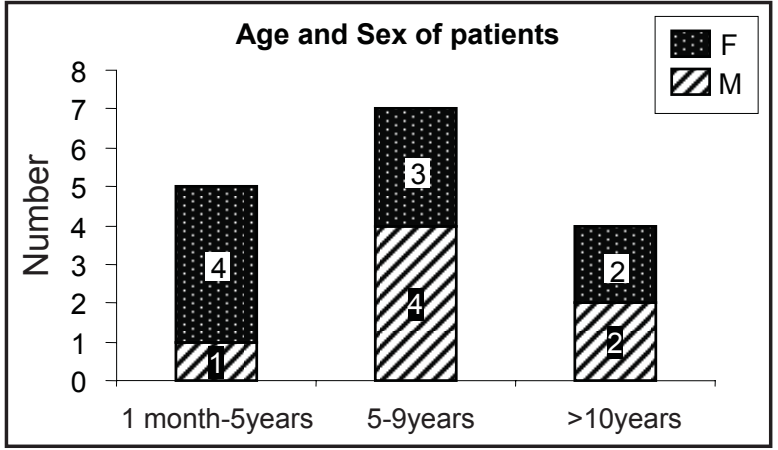


Fig. 4: Showing the various symptoms seen in patients with JE.

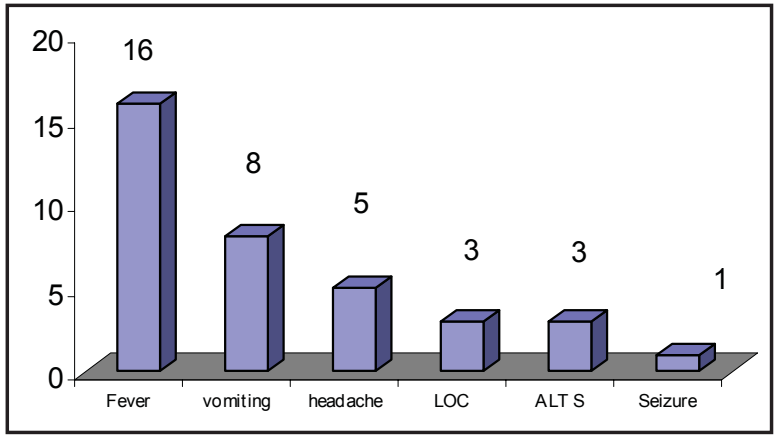

Fig. 5: Showing JE cases in different months of the year.

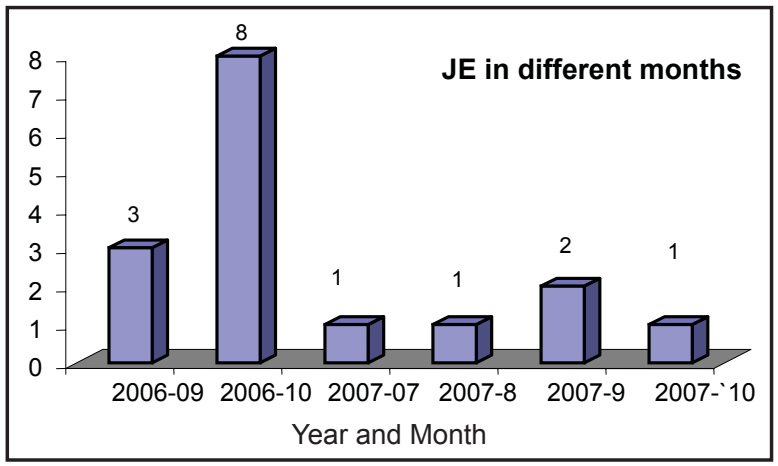

\section{Discussion}

Japanese encephalitis is demonstrated to be a significant public health problem in Nepal and throughout Asia. JE case is considered to have elevated temperature over $38^{\circ} \mathrm{C}$ along with altered consciousness and is generally confirmed serologically by findings of specific anti JE Ig M in the cerebrospinal fluid. This disease primarily affects children between the ages of one and fifteen year's .Of those who contract the disease; approximately, Seventy percent either die or are left with a long term neurological disability. JE vaccines existed for a long time, however due to cost and unstable supply; they have not been able to meet the needs of developing country health systems. As a result, sixty eight (68) percent of babies born in the poorest countries of Asia are at risk for JE. ${ }^{19}$ The disease presents with a prodromal stage, an acute encephalitic stage with coma, convulsions and variable deficits and a convalescent stage. In view of the high mortality and severe sequelae which often leaves behind highly dependent and dependent and disabled survivors, the diseases is assuming great importance. ${ }^{20}$

Japanese Encephalitis is demonstrated to be a significant public health problem throughout Asia. The geographical distribution of JE has expanded over the years to include all of Southeast Asia and most of the Indian Subcontinent. ${ }^{21}$ Epidemics of JE have spread across all of Southeast Asia and were reported for the first time in Nepal in the late1978 and are now endemic in the southern lowlands, probably as a result of steady rise in population density, deforestation and increasing irrigation of agricultural areas ${ }^{22,23}$.Most infections with JE virus cause no symptoms and $<0.1 \%$ develop severe Encephalitis ${ }^{24 .}$

In this study serologically confirmed JE was found in $17.7 \%$ cases. Diagnosis of disease was mostly based on detection of presence of JEV-specific IgM antibodies. A . Rayamajhi et al in their study found lab confirmed JE in $86.2 \%$ cases in one study ${ }^{21}$ and $61.7 \%$ of cases in another study. ${ }^{25}$ Akiba et al had found lab confirmed JE in $78 \%$ of cases. ${ }^{26}$ Our finding was much less than that found by other authors.

This Study showed $56.25 \%$ male patients in comparison to $43.75 \%$ females. A. Rayamajhi in his study found $69 \%$ male and $31 \%$ females, which is about same as in our study. ${ }^{21}$ Study from South India also showed male to be effected in $58 \%$ cases and female sex in $42 \%$ case. $^{3}$

Seventy five percent patients in this study were under nine years of age. Raghava Potula in his study found $71.2 \%$ patients less than ten years. This data is more or less similar to our study. ${ }^{3}$ A. Rayemajhi et al in their study found $58.4 \%$ patients below nine years of age. Number of children less than nine years were more in our study as compared to the one done by Ajit et al ${ }^{21}$. Neeru Gupta from India found $31.25 \%$ cases less than five years of age, more than $4 / 5^{\text {th }}$ of the cases $(84 \%)$ were between one to twelve years of age. ${ }^{27}$

Our study showed that fever was the most common symptom of JE and was found in $100 \%$ cases, the other symptoms seen in this disease were headache $(31.25 \%)$, vomiting $(50.0 \%)$, seizure $(6.25 \%)$, altered sensorium $(18.75 \%)$ and unconsciousness (18.75\%). Ajit et al in their study found Headache in $54.2 \%$ cases, vomiting in $50 \%$ cases and seizures in $58 \%$ cases following symptoms. ${ }^{21}$ Neeru Gupta et al found fever in $100 \%$ cases, vomiting in $41.4 \%$, headache $10.3 \%$ cases and convulsions in $51.7 \%$ cases. ${ }^{27}$ Findings of our study are similar with findings of other authors..

Present study showed most of the cases appeared soon after Monsoon, i.e. during August and September months. Eight seven point sixty percent cases were seen during these two months. Study done by Bista B.M showed, upsurge of cases take place after the rainy season (monsoon). According to his study, cases start to appear in the month of April-May and reach its peak during late August to early September and start to decline from October. ${ }^{11}$ One study from India gives similar report as have maximum no of patients with Encephalitis during the month of July., August and September ${ }^{27}$. So findings of our study go along with other's findings in relation to upsurge of disease.

During the eighteen months of study, two patients died, hence the mortality rate being $12.5 \%$. Study done 
at BP Koirala Institute of Health Sciences showed 8.3 \% mortality rate ${ }^{25}$ and report from Indonesia showed, Case fatality rate of $10 \% .{ }^{28}$ Similarly Akiba T and Kumar R in their studies found case fatality rate of $13.2 \%{ }^{26}$ and $20-50 \%$ simultaneously. ${ }^{29}$ All these findings are similar to our findings. Besides these, our results are consistent with previous studies done in Nepal and the Philippines. Studies from India and Thailand found JE to account for a higher proportion of deaths.

\section{Conclusion}

Japanese encephalitis is a serious public health problem with significant mortality in children. Female patients suffered more than males during early ages of life. Fevers, headache, vomiting and altered sensorium were common symptoms of the diseases. Most cases were seen during summer season (August to October). Case fatality rate was eleven percent. Serogical test is confirmative.

\section{Acknowledgement}

We would like to thank Mr. Macha Bhai Shakya, Liaison officer, Patan Hospital for helping us while preparing this article.

\section{References}

1. Japanese Encephalitis: World Organization for animal Health (OIE), June 3, 2003; 1-3.

2. Kabilan Lolita et al.Japanese Encephalitis in India: An overview. The Indian Journal of Pediatrics 2004; 71: 609-615.

3. Potula R, Badrinath $\mathrm{S}$ et al. Japanese Encephalitis in and around Pondicherry, South India: A Clinical Appraisal and Prognostic Indicators of the outcome. J Trop Pediatrics 2003; 49:48-53

4. Tirounourougane SV, Raghava P, Srinivasan S. Japanese viral Encephalitis. Postgraduate Medical Journal 2002; 78:205-15.

5. Pond WL, Russ SB, Lancaster WF, Audy JR, Smade. Japanese Encephalitis in Malaya. I. Distribution of Neutralization Antibodies in Man and Animal. Am J Hyg 1954 ;59:17-25

6. Banerjee K. Emerging Viral Infections with Special References to India. Indian $J$ Med Res 1996; 103:177-200.

7. Srey VH, Sadones $\mathrm{H}$ et al. Etiology of Encephalitis Syndrome among Hospitalized Children and Adults in Takeo, Cambodia 1999-2000. Am J Trop Med Hyg 2002;66(2): 200-207

8. Ayukawa R, Fujimoto H, Ayabe $\mathrm{M}$ et al. An unexpected outbreak of JE in the Chugoku district of Japan,2002.Jpn J infec Dis 2002;57:63-66

9. Zimmerman MD, Scott RM et al. An outbreak of Japanese Encephalitis in Kathmandu, Nepal. Am J Trop Med Hyg 1997; 57:283-4.
10. Kumar R, Mathur A, Sharma S et al. Clinical Features and Prognostic Indicators of Japanese encephalitis in Children in Lucknow(India). Indian J Med Res.1990;91:321-7

11. Bista MB, Shrestha JM. Epidemiological Situation of Japanese Encephalitis in Nepal. JNMA.2005 Apr-Jun; 44(158):51-6.

12. Jacobson J, Sivalenka S. Japanese Encephalitis Globally and in India. Indian J PH.2004 AprJun;48(2):49-56

13. Kabilan L, Edwin N, Balashankar S, Miranda D. Japanese Encephalitis among Paediatric patients with Acute Encephalitis Syndrome in Tamil Nadu, India. Trans Roy Soc Trop Med and Hyg 2000; 94 : 157-158.

14. Tiroumourougane SV, Raghava P, Srinivasan S, Badrinath. Management parameters affecting the outcome of Japanese Encephalitis. J Trop Pediatr 2003; 49(3): 153-156.

15. Raghava P, Badrinath S, Srinivasan S. Japanese Encephalitis in and around Pondicherry, South India: A Clinical and Prognostic Indicator for the Outcome. J Trop Pediatr 2003; 49(1): 48-53

16. Halstead SB. Arboviruses of the Pacific and Southeast Asia. In Feigin RD, Cherry JD, eds. Textbook of Pediatric Infectious Diseases, 3rd edn. Philadelphia, PA: WB Saunders. 1992; 1468-1475

17. Huy B, Tu H, Luan T, Lindquist R. Early Mental and Neurological Sequelae after Japanese B Encephalitis. Am J Trop Med Hyg 1994; 25: 549553.

18. Philip Samuel P. Japanese encephalitis in India with Special Reference to the Surveillance Tools and Control Measures. Recent Trends in Combating Mosquitoes 2000; 240-252. Proceeding of Loyola College, Chennai, October 3-4, 2000

19. Jacobson J, Sivalenka S. Japanese Encephalitis Globally and in India. Indian $J$ Public Health. 2004 Apr-Jun; 48(2):49-56

20. Sehgel A, Dutta AK Changing perspectives in Japanese Encephalitis in India. Trop Doc. 2005 Apr; 35(2):122

21. Ajit Rayamajhi et al. Clinico-laboratory Profile and outcome of Japanese Encephalitis in Nepali Children. Annals of Tropical Pediatrics 2006; 26,293-301

22. Bista MB. Arboviral encephalitis. In: Bista MB ed. Infectious diseases in Nepal, $1^{\text {st }}$ Ed. Kathmandu: Epidemiology and Disease Control Division, Department of Health services, Ministry of Health and World Health organization. 2001; 1-29 
23. Ogawa S, Shrestha MP, Rai SK, et al. Serological and Virological Studies of Japanese Encephalitis in Terai region of Nepal. Southeast Asian J Trop Med Public Health1992; 23:37-43

24. Chaudhary N, Shaw BP, Mondal KC, Maity CR. Epidemiology of Japanese Encephalitis. Indian Paeditr 1992; 29:861-5.

25. Ajit Rayamajhi et al. Study of Japanese Encephalitis and other Viral Encephalitis In Nepali Children. Paediatrics International. 2007;49,978-984.

26. Akiba T, Osaka K, Tang et al. Analysis of Japanese Encephalitis Epidemic in Western Nepal in 1997. Epidemiology Infect.2001 Feb; 126(1):81-8
27. Gupta Neeru et al. Bellary, India Achieves Negligible Case Fatality due to JE Investigation in 2004. Ind J Pediatr 2008; 75: 31-37

28. Komang Kari et al. A Hospital Based Surveillance for Japanese Encephalitis in Indonesia. BMC Med.2006; 4:8

29. Kumar R. Viral Encephalitis of Public Health Significance in India: Current Status. Indian $J$ Pediatr.1999,Jan -Feb;66(1):73-83. 